\title{
PARAMENTAÇÃO CIRÚRGICA: AVALIAÇÃO DE SUA ADEQUAÇÃO PARA A PREVENÇÃ̄O DE RISCOS BIOLÓGICOS EM CIRURGIAS. PARTE I: A UTILIZAÇÃO DURANTE AS CIRURGIAS*
}

\author{
SURGICAL SCRUB: EVALUATING ITS ADEQUATION FOR PREVENTING \\ BIOLOGICAL RISKS IN SURGERIES. PART I: THE USE IN SURGERIES
}

\author{
Marielen Silva de Oliveira Paz *** \\ Rúbia Aparecida Lacerda** \\ Cibele Estanislau da C. Monteiro *** \\ Viviane Peres da Conceição***
}

PAZ, M.S. de O. et al. Paramentação cirúrgica: avaliação de sua adequação para a prevenção de riscos biológicos em cirurgias. Parte I: a utilização durante as cirurgias. Rev.Esc.Enf.USP, v. 34, n. 1, p. 108 -117, mar. 2000.

\section{RESUMO}

O estudo elaborou critérios para uso adequado da paramentação cirúrgica e avaliou, por observação sistematizada, o desempenho da equipe cirúrgica durante a cirurgia. O procedimento adotado foi válido, permitindo reconhecer aspectos positivos e problemáticos. A exceção do óculos protetor, todos os outros componentes da paramentação foram utilizados. A questão se situou na forma de utilização, onde gorros e máscaras foram mais problemáticos entre anestesistas e circulantes; avental e luvas entre cirurgiões e, principalmente, instrumentadores.

PALAVRAS-CHAVES: Controle de infecções. Infecção hospitalar. Roupa de proteção.

\begin{abstract}
The study elaborated approaches for adequated use of the surgical scrub and it evaluated, through systematized observation, the performance of the surgical team during the surgery. The adopted procedure was very valid, allowing to recognize positive and problematic aspects. Except the protecting glasses, all the other components of the surgical scrub were used. The subject located in the use form, where caps and masks were more problematic among anesthesists and circulate nurses; apron and gloves among surgeons and, mainly, scrub nurses.
\end{abstract}

KEYWORDS: Infection control. Cross infection. Protective clothing.

\section{INTRODUÇÃO}

Inicialmente, o uso da paramentação cirúrgica objetivava principalmente a proteção dos clientes contra contaminação do sítio cirúrgico por microorganismos liberados pelo ambiente, pessoas, materiais e equipamentos em sala de operações (SO). Com a ênfase atual na proteção também dos profissionais contra exposição ao sangue e outros fluidos, essas barreiras ganharam nova dimensão, sendo objeto de inúmeras investigações para a sua melhor qualificação.
Uma avaliação da paramentação deve começar pelas respostas às questões: a) o que é paramentação cirúrgica? b) a paramentação é realmente necessária para prevenir riscos biológicos em cirurgia? c) se ela é necessária, quais os critérios para considerar seu uso adequado?

A paramentação é um conjunto de barreiras contra a invasão de microorganismos nos sítios cirúrgicos dos clientes e para proteção de exposição dos profissionais a sangue e outros fluidos orgânicos

\footnotetext{
* $\quad$ Pesquisa realizada com o apoio da FAPESP (Fundação de Amparo à Pesquisa do Estado de São

** Paulo) Enfermeira. Professora doutora da Escola de Enfermagem da USP

*** Enfermeiras. Graduadas pela Escola de Enfermagem da USP
} 
de clientes. Constituem-se de: propés, uniforme privativo (jaleco e calça), gorro, máscara, avental, luvas, campos da área cirúrgica e protetor ocular.

$\mathrm{Na}$ segunda questão, há que se discutir antes, sobre a ocorrência de infecção em cirurgias.

A infecção no cliente caracteriza-se pela sua variedade, quanto aos agentes etiológicos e formas de transmissão ${ }^{23}$. A infecção do sítio cirúrgico é uma das causas mais comuns de infecção hospitalar. Apesar de se manifestar após a cirurgia, a maior chance de contaminação é durante a cirurgia, já que os sitios anatômicos são invadidos por tempo prolongado, com intensa manipulação. Portanto, é durante a cirurgia que o controle de infecção deve ser redobrado 22 .

A maioria das infecções que acometem o cliente são provocadas pela flora humana (endógena). Em SO, ela origina-se do próprio cliente e dos profissionais, além do ar ambiente, materiais e equipamentos contaminados por essa própria flora 22,23 Das formas de transmissão conhecidas12, as que o cliente está mais susceptível são contato, veiculação, goticulas e ar ambiente. As três primeiras já estão comprovadas. Já, a transmissão direta de microorganismos pelo ar ambiente ainda é objeto de intensa discussão. No entanto, não foram encontrados estudos que menosprezem a sua importância, principalmente nos casos de infecção de cirurgias limpas 5, 28, 34,36.

Os riscos nos profissionais também existem. A principal forma de transmissão é o contato com sangue e outros fluidos do cliente. Um resumo da literatura dos últimos anos mostra os seguintes resultados 4,10,15,21,23,24,25,26,27,30,31,33,35,37: cirurgiões são os mais expostos, conforme a especialidade cirúrgica as técnicas utilizadas; os locais mais freqüentes são mãos, seguidos de face, pescoço e mucosas; $1,0 \%$ a $15,0 \%$ correspondem a injúrias percutâneas; a transmissão de HIV é muito baixa (cerca de 0,09\% em contato com mucosa e 0,3\% em percutâneo). Já, risco de transmissão de Hepatite B é cerca de 100 vezes maior do que de HIV.

O reconhecimento dos modos de transmissão em SO para clientes e profissionais

e os riscos decorrentes justificam plenamente a utilização da paramentação. Do lado dos clientes, contendo a liberação dos microorganismos da sua própria flora e evitando sua transmissão por contato, goticulas e ar ambiente nos sitios invadidos. Do lado dos profissionais, evitando o contato de pele e mucosas com o sangue e outros fluidos do cliente.
$\mathrm{Na}$ terceira questão, a paramentação adequada se refere tanto às especificações técnicas de sua confecção, quanto à forma como é utilizada. Estudos recentes têm se voltado principalmente para a discussão da primeira questão, ou seja a busca de confecção "ideal" como barreira 1,2,3,6,7,8,9,10,11,13,14,15,16,17,18,19,20,25,26,29,31,32, justificados

pela ausência de padronização para a sua confecção, a qual permite variedade tanto nos tecidos com que são confeccionadas, quanto nos seus modelos e dificultando assim, sua confiabilidade como barreira para as funções a que se destinam.

Essa parte do estudo investigou a forma como a paramentação está sendo utilizada*, tendo como objetivos: a) elaborar critérios de avaliação de adequação da utilização da paramentação cirúrgica; b) avaliar o uso da paramentação durante todo o ato operatório.

\section{METODOLOGIA}

Pesquisa exploratória, com investigação pautada em entrevista e observação sistematizada. A população foi composta por membros de equipes cirúrgicas. Os locais do estudo foram salas de operações de 14 hospitais do Município de São Paulo (que responderam que concordavam em participar, após correspondência enviada a todos os hospitais a partir de 200 leitos), sendo 4 privados e 10 públicos, 8 de médio porte e $\mathbf{6}$ de grande porte; 6 realizavam de $100-200$ cirurgias/ mês, 4 de 201 a 500 cirurgias; 4 mais de 500 cirurgias (Anexo 1- parte A).

$\mathrm{Na}$ definição da amostra, os dados foram coletados de uma cirurgia de cada hospital através de observação direta: dos profissionais que concordaram previamente em participar da pesquisa, totalizando 79 pessoas (30 cirurgiões, 10 instrumentadores, 19 anestesistas e 21 circulantes), quanto ao uso da paramentação durante todo o ato cirúrgico (Anexo 1- parte B), o qual foi classificado em 3 momentos: $1^{\circ}$ ) da degermação e paramentação até o preparo do paciente, materiais e equipamentos; $2^{\circ}$ ) cirurgia propriamente dita (início ao fechamento da incisão); $3^{\circ}$ ) retirada da paramentação. Essa classificação deveu-se a: a) tempos diferentes de participação dos membros da equipe no ato cirúrgico; b) etapas distintas para utilização pela equipe cirúrgica, de alguns componentes da paramentação; c) possibilidade de iniciarem o uso de modo inadequado e corrigirem no decorrer da cirurgia e/ou vice-versa.

* Para a análise e avaliação das especificações técnicas da paramentação cirúrgica, foi realizada outra pesquisa, apresentada como parte II deste estudo 


\section{CRITÉRIOS PARA AVALIAÇÃO DO USO DA PARAMENTAÇÃO CIRÚRGICA}

O uso adequado da paramentação inicia-se pela seqüência de sua colocação, quando a touca, seguida do jaleco e da calça comprida, são colocados no vestiário. O propé é calçado ao sair do vestiário ou da área comum de pessoas sem propés. A máscara, transportada no bolso do uniforme (e não no pescoço, para evitar sua colonização antecipada), é usada por todos os membros da equipe apenas para entrar na área restrita (SO) ${ }^{9,18}$

Os outros componentes da paramentação são colocados quando se inicia a cirurgia, conforme a função de cada categoria e o momento do ato operatório. Os óculos são usados em toda a cirurgia por .cirurgiões e instrumentadores; em alguns momentos, por anestesistas e circulantes (em procedimentos com risco de respingos de substâncias orgânicas como indução anestésica, aspiração, etc.). O avental é vestido pelos cirurgiões e instrumentadores na So, após a degermação e secagem das mãos, permanecendo até o final das cirurgias. As luvas são calçadas pelos cirurgiões e instrumentadores, em seguida aos aventais. Os anestesistas e circulantes calçam luvas em situações específicas (realização de procedimentos assépticos ou em que há riscos de exposição ocupacional com substâncias orgânicas dos clientes). Tais componentes não devem ser usados fora da área restrita. Jóias não são recomendadas ${ }^{1,2}$. A seguir, são tecidos alguns comentários por componentes da paramentação.

Touca: barreira de proteção contra microorganismos do cabelo e couro cabeludo, em tamanho adequado para a cobertura total do cabelo e sem solução de continuidade (furos, rasgos) para não "quebrar" a barreira asséptica. Por ser apenas limpa, não pode tocar em superficies estéreis.

Uniforme privativo: evita liberação de microorganismos da pele, tronco e membros, fornecido em tamanhos que atendam os diferentes manequins dos usuários. O jaleco precisa cobrir todo o tronco, do final do pescoço até o início da pélvis. Devido riscos de contato dos braços com fluidos orgânicos, deve ter manga mais longa ${ }^{2}$. Isso também protege contra a liberação de microorganismos das axilas. A calça precisa cobrir totalmente os membros inferiores, protegendo o trabalhador e também evitando a liberação ao ambiente da flora de suas pernas e períneo. AYLIFFE et $\mathrm{al}^{2}$ sugerem calças com fechamento nos tornozelos como mais adequada para impedir a liberação dessa flora. Por ser apenas limpo, o uniforme não pode tocar em superficies estéreis.

Propé e/ou sapato privativo: justificado na prevenção de contaminação do chão de áreas críticas por microorganismos que são carreados nas solas dos sapatos e podem ser liberados ao ambiente. Porém, sua eficácia no controle de infecções tem sido questionada em vários estudos, cujo resumo de suas conclusões é apresentado a seguir 3,6,8,11,12,13,17,18,32:

- diferença não significativa de contaminação do chão entre calçados comuns e propés;

- não é habitual a troca de propés, mesmo após pisotear secreções orgânicas presentes no chão, que se disseminam por todas as áreas do centro cirúrgico;

- o ato de caminhar é a mais importante causa da dispersão bacteriana do chão do que o tipo de cobertura de sapatos utilizado;

- microorganismos do chão dificilmente se dissipam ao ar ambiente e não são aqueles causadores de infecção do sítio cirúrgico;

- a chance de contaminação de uma ferida com microorganismos oriundos do chão se dá antes pela sua veiculação e por contato do que pela sua disseminação do chão para o ar ambiente.

Mesmo assim, coberturas de sapatos ou sapatos privativos continuam sendo recomendados nos EUA para proteção do trabalhador contra contaminação com fluidos 9 . Entretanto, há que se atentar também para a sua eficácia como barreira, uma vez que esses dispositivos atualmente utilizados (tamancos com orificios para transpiração e propés) não evitam ferimento dos pés com objeto pérfuro-cortante. Nesse caso, o ideal seriam sapatos privativos fechados, pois os modelos anterioremente citados não contribuem para o controle de infecções e ainda determinam custos desnecessários. Outros estudos também discutem a eficiência dos propés como barreiras, de acordo com o tipo de tecido com que são confeccionados e serão apresentados na parte II deste estudo. Diante do exposto, o uso de coberturas de sapatos não será avaliado neste estudo.

Máscara cirúrgica ou protetor respiratório: evita a liberação de microorganismos oriundos do nariz e da boca dos profissionais, protegendo o cliente de contaminação na incisão cirúrgica. Do lado do profissional, protege suas mucosas de respingos de sangue e outros fluidos do cliente. Para isso, a máscara deve apresentar capacidade minima de filtração por certo período de tempo e, nesse aspecto, há dificuldades para se determinar as suas características ideais de confecção, cuja discussão é apresentada na parte II deste estudo $2,7,9,18,19,20$ Nessa primeira parte da pesquisa, enfatiza-se que quaisquer protetores respiratórios não têm eficácia alguma se não forem usados corretamente. Isso significa que devem ser colocados por todos que entram e permanecem na SO, com cobertura completa do nariz e da boca, aderindo à pele nas regiões superior, inferior e laterais do rosto 18 .

Estudos citam que as máscaras convencionais apresentam uma vida útil de 2 horas, findas as quais é desejável que sejam trocadas 18 ou mesmo antes, por ocorrência de sujidade ou excesso de umidade. Por serem apenas limpas, não devem entrar em contato com superficies estéreis. 
Avental cirúrgico: evita a liberação de microrganismos oriundos do corpo dos profissionais e a contaminação dos sítios invadidos dos clientes. Com relação ao profissional, protege a pele do corpo da exposição ao sangue e outras substâncias orgânicas do cliente. Na literatura predominam estudos sobre tipos de tecidos para confecção e suas vantagens e desvantagens como barreira microbiana $1,10,29,31,37$, os quais serão discutidos posteriormente, no outro estudo (parte II).

Neste estudo (parte I), a avaliação se referirá à forma de uso, que compreende:

- tamanhos adequados aos diferentes manequins dos usuários, com cobertura completa do tronco (a partir do final do pescoço, membros superiores até o punho e inferiores até os joelhos);

- esterilizados, o que inclui abrir o pacote, desdobrar e vestir o avental tocando somente no lado interno (que ficará em contato com o corpo). O lado externo não deve tocar em superficies não estéreis. É retirado pelo avesso, sendo tocado somente em seu lado interno, dessa vez, para proteger o profissional contra contaminação ocorrida do lado externo.

Protetor ocular: recomendado especialmente para proteção dos trabalhadores. Seu modelo deve permitir aderência à pele circunvizinha, evitando o contato da mucosa ocular com sangue e outros fluidos e impedindo o embaçamento por penetração da expiração.

Luvas cirúrgicas: servem como barreira tanto para proteger o cliente da flora microbiana das mãos da equipe cirúrgica, como para evitar infecção ocupacional pelo contato com sangue do cliente. Para tanto, as preocupações se iniciam com sua qualidade (flexibilidade, impermeabilidade e resistência ao tempo e movimentos cirúrgicos) . Mesmo assim, não estão livres de sofrerem desgastes, furos e rasgos ${ }^{38}$, por decorrência do tempo e do tipo de cirurgia. Uma alternativa é o uso de luvas duplas em cirurgias acima de 1 hora. Porém, queixas vêm sendo apresentadas, pela diminuição da sensibilidade tátil e "aperto" das mãos em demasia 14,26,30,37 Outra alternativa tem sido a recomendação de troca das luvas a cada 2 horas de cirurgia, além de sua observação frequente ${ }^{2}$.

As luvas devem ser usadas com técnica asséptica, para proteção do cliente. O lado externo só pode tocar em superficies estéreis e no sítio cirúrgico. Para proteção do profissional, as luvas devem ser retiradas pelo avesso, sendo tocadas apenas na sua superficie interna.

\section{RESULTADOS E DISCUSSÃO}

Os resultados e a discussão são apresentados por categoria profissional, considerando a utilização de cada componente da paramentação cirúrgica e cada momento do ato cirúrgico. $\mathrm{O}$ número de profissionais nem sempre foi o mesmo nos 3 momentos considerados do ato cirúrgico, já que nem todos iniciaram e terminaram juntos a cirurgia. Assim, o total nas tabelas refere-se ao total de profissionais que estiveram na SO em cada momento do ato cirúrgico e não ao total geral observado por categoria profissional. Para a avaliação, elaboramos, a partir da discussão bibliográfica apresentada, o que segue:

a) adequado: o uso do componente atendeu os critérios de adequação discutidos no item IV do anexo 2 durante os 3 momentos do ato cirúrgico considerado; b) parcialmente adequado: 0 uso atendeu os critérios de adequação, porém isso não ocorreu em todos os 3 momentos do ato cirúrgico; c) inadequado: o uso não atendeu os' critérios de adequação em todos os momentos do ato cirúrgico.

Tabela 1 - Avaliação do uso da paramentação cirúrgica pelos cirurgiões, durante a cirurgia. São Paulo, 1998.

\begin{tabular}{|c|c|c|c|c|c|c|c|c|c|c|c|c|c|c|c|}
\hline \multirow{2}{*}{$\begin{array}{l}\text { AVALIAÇÃO } \\
\text { MOMENTO } \\
\text { COMPON. } \\
\end{array}$} & \multicolumn{3}{|c|}{ ADEQUADO } & \multicolumn{3}{|c|}{ PARC. ADEQ } & \multicolumn{3}{|c|}{ INADEQUADO } & \multicolumn{3}{|c|}{ NÃO UTILIZOU } & \multicolumn{3}{|c|}{ TOTAL } \\
\hline & $1^{\circ}$ & $2^{\circ}$ & $3^{\circ}$ & $1^{\circ}$ & $2^{\circ}$ & $3^{\circ}$ & $1^{\circ}$ & $2^{\circ}$ & $3^{\circ}$ & & & $3^{\circ}$ & & 上 & $3^{\circ}$ \\
\hline $\begin{array}{l}\text { PROT. } \quad \mathbf{N}^{\circ} \\
\text { OCULAR \% }\end{array}$ & $\begin{array}{c}07 \\
25,0\end{array}$ & , & 0,0 & $\begin{array}{r}0 \\
0,0\end{array}$ & $\begin{array}{r}0 \\
0,0\end{array}$ & 0,0 & \begin{tabular}{|c}
0 \\
0,0
\end{tabular} & 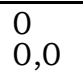 & ( & & & & $\begin{array}{l}28 \\
100\end{array}$ & 00 , & 00 \\
\hline $\begin{array}{ll}\text { UNIF. } & \mathbf{N}^{\circ} . \\
\text { PRIV. } & \%\end{array}$ & $\begin{array}{c}27 \\
96,4\end{array}$ & $\begin{array}{c}29 \\
96,7\end{array}$ & $\begin{array}{r}20 \\
100\end{array}$ & $\begin{array}{r}0 \\
0,0\end{array}$ & $\begin{array}{r}0 \\
0,0\end{array}$ & $\begin{array}{l}0 \\
0,0\end{array}$ & $\begin{array}{l}01 \\
3,6\end{array}$ & & $\begin{array}{c}0 \\
0,0\end{array}$ & $\begin{array}{l}0 \\
0,\end{array}$ & 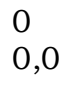 & $\begin{array}{l}0 \\
, 0\end{array}$ & $\begin{array}{r}28 \\
100\end{array}$ & $\begin{array}{r}30 \\
100\end{array}$ & $\begin{array}{r}20 \\
100\end{array}$ \\
\hline $\begin{array}{r}\text { MÁsC. } \mathbf{N}^{\circ} \\
\%\end{array}$ & $\begin{array}{c}22 \\
78,6\end{array}$ & $\begin{array}{r}22 \\
73,3\end{array}$ & $\begin{array}{r}13 \\
65,0\end{array}$ & $\begin{array}{r}0 \\
0,0\end{array}$ & $\begin{array}{r}0 \\
0,0\end{array}$ & $\begin{array}{l}0 \\
0,0\end{array}$ & $\begin{array}{c}06 \\
21,4\end{array}$ & & $\begin{array}{r}07 \\
35,0\end{array}$ & 0 & & & $\begin{array}{r}28 \\
100\end{array}$ & $\begin{array}{r}30 \\
100\end{array}$ & $\begin{array}{r}20 \\
100\end{array}$ \\
\hline GORRO N & 18 & 21 & 10 & 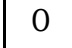 & 0 & 0 & 10 & 09 & 07 & 0 & 0 & 0 & 2 & 5 & 20 \\
\hline & $\begin{array}{c}64,3 \\
16\end{array}$ & 2 & $\begin{array}{c}65,0 \\
17\end{array}$ & $\begin{array}{r}0,0 \\
0\end{array}$ & $\begin{array}{r}0,0 \\
0\end{array}$ & $\begin{array}{l}0,0 \\
0\end{array}$ & $\begin{array}{l}35,7 \\
02\end{array}$ & & $\begin{array}{r}35,0 \\
02\end{array}$ & & & & $\begin{array}{r}100 \\
28\end{array}$ & $\begin{array}{r}100 \\
30\end{array}$ & $\begin{array}{r}00 \\
20\end{array}$ \\
\hline RG \% & 57,2 & 80,0 & 85,0 & 0,0 & 0,0 & 0,0 & 1,1 & $10, \pi$ & $10, \pi$ & , & & 50 & 100 & 10 & 100 \\
\hline $\begin{array}{l}\text { AVENTAL N } \\
\text { CIRÚRG. \% }\end{array}$ & 50,0 & 50,0 & 55,0 & $\begin{array}{c}0 \\
0,0\end{array}$ & $\begin{array}{r}0 \\
0,0\end{array}$ & $\begin{array}{l}0 \\
0,0\end{array}$ & $\begin{array}{c}12 \\
42,9\end{array}$ & 46,7 & 40,0 & 7,1 & & 5,0 & 100 & 100, & 100 \\
\hline RG. \% & $\begin{array}{c}16 \\
57,2\end{array}$ & 60,0 & $\begin{array}{r}12 \\
60,0\end{array}$ & $\begin{array}{c}0 \\
0,0\end{array}$ & $\begin{array}{r}0 \\
0,0\end{array}$ & 0,0 & 35,7 & 36,7 & $\begin{array}{r}08 \\
40,0\end{array}$ & $\begin{array}{l}02 \\
7,2\end{array}$ & 3 & 0,0 & 100, & 100 & 00 \\
\hline
\end{tabular}


Os cirurgiões utilizam a paramentação completa. Porém, isso nem sempre ocorreu, pois a luva, o avental, o campo e o protetor ocular, não foram utilizados por todos.

A não utilização do protetor ocular por quase todos sugere que esta prática ainda não está incorporada, justamente, um item de proteção individual. Outras razões podem estar coincidindo com achados em estudos científicos, como diminuição visual do campo operatório, embaçamento pelo escape de respiração dentro do visor, ausência de risco de respingamento de fluidos em determinadas cirurgias, crenças em riscos etc.

A não utilização de luvas e avental por alguns deveu-se ao fato de que nem todos participaram diretamente do ato cirúrgico. A não utilização do campo foi maior no $1^{\circ}$ momento (corresponde à montagem do campo operatório) porque, com grande frequência, é feito pelo instrumentador. Os maiores índices de adequação foram o uniforme privativo, o campo e a máscara. Já, uso inadequado significativo ocorreu com avental cirúrgico, luvas e gorros.

A inexistência de avaliação como parcialmente adequada revela que as falhas e os acertos iniciaram e terminaram sem terem sido corrigidas. As situações que determinaram avaliação inadequada foram:

- uniforme privativo: calças curtas.

- gorros: mechas de cabelo soltas.

- máscaras: não utilização dentro da SO; abertura nas laterais; contaminação com sangue.

- campos: contato com o chão ao serem abertos; contato com outras superficies não estéreis; má fixação permitindo o contato do cirurgião com pele que não do sítio cirúrgico do cliente.

- avental: contato do seu lado estéril com mãos não enluvadas; contato das mãos sem luvas com regiões contaminadas do avental no momento de sua retirada.

- luvas : na colocação, o lado externo (que deve permanecer estéril) foi tocado com as mãos; na retirada, as mãos tocaram no lado externo (agora contaminada pela cirurgia).

- protetores oculares:_não houve

inadequação para aqueles que os utilizaram.

Tabela 2 - Avaliação do uso da paramentação cirúrgica pelos instrumentadores, durante a cirurgia. São Paulo, 1998.

\begin{tabular}{|c|c|c|c|c|c|c|c|c|c|c|c|c|c|c|c|}
\hline \multirow{2}{*}{$\begin{array}{l}\text { AVAL. } \\
\text { MOMENTO } \\
\text { COMPON. }\end{array}$} & \multicolumn{3}{|c|}{ ADEQUADO } & \multicolumn{3}{|c|}{ PARC. ADEQ. } & \multicolumn{3}{|c|}{ INADEQUADO } & \multicolumn{3}{|c|}{ NÃO UTILIZOU } & \multicolumn{3}{|c|}{ TOTAL } \\
\hline & $1^{\circ}$ & $\mathbf{2}^{\circ}$ & $3^{\circ}$ & $1^{\circ}$ & $2^{\circ}$ & $3^{\circ}$ & $1^{\circ}$ & $2^{\circ}$ & $\mathbf{3}^{\circ}$ & $1^{\circ}$ & $2^{\circ}$ & $3^{\circ}$ & $1^{\circ}$ & $2^{\circ}$ & $3^{\circ}$ \\
\hline PROT. $\mathbf{N}^{\circ}$ & 0 & 0 & 0 & 0 & 0 & 0 & 0 & 0 & 0 & 09 & 10 & 09 & 09 & 10 & 09 \\
\hline CUL. \% & 0,0 & 0,0 & 0,0 & 0,0 & 0,0 & 0,0 & 0,0 & 0,0 & 0,0 & 100 & 100 & 100, & 100, & 100, & 100 \\
\hline UNIF. $\quad \mathbf{N}^{\circ}$ & 08 & 09 & 08 & 0 & 0 & 0 & 01 & 01 & 01 & 0 & 0 & 0 & 09 & 10 & 09 \\
\hline PRIV. \% & 90,0 & 90,0 & 90,0 & 0,0 & 0,0 & 0,0 & 10,0 & 10,0 & 10,0 & 0,0 & 0,0 & 0 & 100, & 100, &, 100 \\
\hline MÁSC. $\mathbf{N}^{\circ}$ & $\begin{array}{r}07 \\
77,8\end{array}$ & $\begin{array}{l}06 \\
60,0\end{array}$ & $\begin{array}{l}04 \\
44,5\end{array}$ & $\begin{array}{l}0 \\
0,0\end{array}$ & $\begin{array}{l}01 \\
10,0\end{array}$ & $\begin{array}{r}0 \\
0,0\end{array}$ & $\begin{array}{r}02 \\
22,2\end{array}$ & $\begin{array}{l}03 \\
30,0\end{array}$ & $\begin{array}{l}05 \\
55,5\end{array}$ & $\begin{array}{r}0 \\
0,0\end{array}$ & $\begin{array}{l}0 \\
0,0\end{array}$ & $\begin{array}{l}0 \\
0,0\end{array}$ & $\begin{array}{l}09 \\
100\end{array}$ & $\begin{array}{r}10 \\
100\end{array}$ & $\begin{array}{r}09 \\
100\end{array}$ \\
\hline GORRO $\mathbf{N}^{\circ}$ & $\begin{array}{r}07 \\
77,8\end{array}$ & $\begin{array}{l}07 \\
70,0\end{array}$ & $\begin{array}{l}07 \\
77,8\end{array}$ & $\begin{array}{l}0 \\
0,0\end{array}$ & $\begin{array}{l}0 \\
0,0\end{array}$ & $\begin{array}{r}0 \\
0,0\end{array}$ & $\begin{array}{r}02 \\
22,2\end{array}$ & 30,0 & $\begin{array}{l}02 \\
22,2\end{array}$ & $\begin{array}{r}0 \\
0,0\end{array}$ & $\begin{array}{l}0 \\
0,0\end{array}$ & $\begin{array}{l}0 \\
0,0\end{array}$ & $\begin{array}{l}09 \\
100\end{array}$ & $\begin{array}{r}10 \\
100\end{array}$ & $\begin{array}{r}09 \\
100\end{array}$ \\
\hline $\begin{array}{l}\text { CAMPO N} \\
\text { CIRÚR. } \%\end{array}$ & $\begin{array}{r}07 \\
77,8\end{array}$ & $\begin{array}{l}08 \\
80,0\end{array}$ & 77,8 & $\begin{array}{l}0 \\
0,0\end{array}$ & $\begin{array}{l}0 \\
0,0\end{array}$ & $\begin{array}{r}0 \\
0,0\end{array}$ & $\begin{array}{r}02 \\
22,2\end{array}$ & 20,0 & $\begin{array}{l}02 \\
22,2\end{array}$ & $\begin{array}{r}0 \\
0,0\end{array}$ & 0,0 & 0,0 & $\begin{array}{l}09 \\
100\end{array}$ & $\begin{array}{r}10 \\
100\end{array}$ & $\begin{array}{r}09 \\
100\end{array}$ \\
\hline $\begin{array}{ll}\text { AV. } & \mathbf{N}^{\circ} \\
\text { CIRÚR. } & \%\end{array}$ & $\begin{array}{r}03 \\
33,3\end{array}$ & $\begin{array}{l}03 \\
30,0\end{array}$ & $\begin{array}{l}06 \\
66,7\end{array}$ & $\begin{array}{l}0 \\
0,0\end{array}$ & $\begin{array}{l}0 \\
0,0\end{array}$ & $\begin{array}{r}0 \\
0,0\end{array}$ & $\begin{array}{r}06 \\
66,7\end{array}$ & $\begin{array}{l}07 \\
70,0\end{array}$ & $\begin{array}{l}03 \\
33,3\end{array}$ & $\begin{array}{r}0 \\
0,0\end{array}$ & $\begin{array}{l}0 \\
0,0\end{array}$ & $\begin{array}{l}0 \\
0,0\end{array}$ & $\begin{array}{l}09 \\
100\end{array}$ & $\begin{array}{r}10 \\
100\end{array}$ & $\begin{array}{r}09 \\
100\end{array}$ \\
\hline $\begin{array}{l}\text { LUVA } \mathbf{N}^{\circ} \\
\text { CIRÚRG. \% }\end{array}$ & $\begin{array}{r}03 \\
33,3 \\
\end{array}$ & $\begin{array}{l}04 \\
40,0 \\
\end{array}$ & 66,7 & $\begin{array}{l}0 \\
0,0 \\
\end{array}$ & $\begin{array}{l}0 \\
0,0 \\
\end{array}$ & $\begin{array}{r}0 \\
0,0 \\
\end{array}$ & $\begin{array}{r}06 \\
66,7 \\
\end{array}$ & 60,0 & 33,3 & $\begin{array}{r}0 \\
0,0 \\
\end{array}$ & 0,0 & 0,0 & $\begin{array}{l}09 \\
100\end{array}$ & $\begin{array}{r}10 \\
100\end{array}$ & $\begin{array}{r}09 \\
100 \\
\end{array}$ \\
\hline
\end{tabular}

Como os cirurgiões, os instrumentadores devem utilizar a paramentação completa. Porém, nenhum deles usou óculos de proteção.

Os componentes com maior índice de adequação durante toda a cirurgia foram o uniforme privativo, o campo e o gorro. Já, a máscara, apesar de ter iniciado com um bom índice de adequação no $1^{\circ}$ momento, foi caindo para parcialmente adequada no $2^{\circ} \mathrm{e}$ inadequada no $3^{\circ}$. Alto indice de inadequação foi observado no uso do avental e da luva, principalmente nos $1^{\circ}$ e $3^{\circ}$ momentos. Isso sugere que a técnica de colocação e a retirada de ambos ao final da cirurgia é problemática.

A existência de somente uma avaliação como parcialmente adequada (máscara) indica que as falhas no uso da paramentação não foram corrigidas pelos instrumentadores durante toda a cirurgia. Seguem, as situações que determinaram a avaliação inadequada de cada componente:

- uniforme privativo :_calças curtas.

- gorros : mechas do cabelo soltas. 
- máscara :_abertura nas laterais.

- campo:_contato com o chão e com o uniforme privativo ao manuseá-los.

- avental :_na colocação, contato das mãos sem luvas e com terceiros com o lado externo do avental (que deve permanecer estéril); durante a cirurgia, não fechamento completo da parte posterior das costas; na retirada, contato das mãos sem luvas ou em terceiros com região contaminada do avental.

- luvas : na colocação, o par de luvas foi segurado com as mãos, tocando o seu lado estéril; na retirada, contato das mãos com região contaminada da luva.

Tabela 3 - Avaliação do uso da paramentação cirúrgica pelos anestesistas, durante a cirurgia. São Paulo, 1998.

\begin{tabular}{|c|c|c|c|c|c|c|c|c|c|c|c|c|c|c|c|}
\hline \multirow{2}{*}{$\begin{array}{l}\text { AVAL. } \\
\text { MOMENTO } \\
\text { COMPON. }\end{array}$} & \multicolumn{3}{|c|}{ ADEQUADO } & \multicolumn{3}{|c|}{ PARC. ADEQ. } & \multicolumn{3}{|c|}{ INADEQUADO } & \multicolumn{3}{|c|}{ N.UTILIZOU } & \multicolumn{3}{|c|}{ TOTAL } \\
\hline & $1^{\circ}$ & $2^{\circ}$ & $3^{\circ}$ & 1 & 2 & ${ }^{\circ} 3^{\circ}$ & $1^{\circ}$ & $2^{\circ}$ & $3^{\circ}$ & 1 & $2^{\circ}$ & $3^{\circ}$ & $1^{\circ}$ & $2^{\circ}$ & $3^{\circ}$ \\
\hline UNIF. $\quad \mathbf{N}^{\circ}$ & 19 & 16 & 14 & 0 & 0 & 0 & 0 & 0 & 0 & 0 & 0 & 0 & 19 & 16 & 14 \\
\hline $\begin{array}{l}\text { PRIV. \% } \\
\text { MÁSC. N }\end{array}$ & $\begin{array}{l}100 \\
05\end{array}$ & $\begin{array}{r}10,0 \\
04\end{array}$ & $\begin{array}{r}100 \\
04\end{array}$ & $\begin{array}{l}0,0 \\
03\end{array}$ & $\begin{array}{l}0,0 \\
03\end{array}$ & $\begin{array}{l}0,0 \\
04\end{array}$ & $\begin{array}{l}0,0 \\
11\end{array}$ & $\begin{array}{l}0,0 \\
09\end{array}$ & $\begin{array}{l}0,0 \\
06\end{array}$ & $\begin{array}{r}0,0 \\
0\end{array}$ & $\begin{array}{r}0,0 \\
0\end{array}$ & $\begin{array}{r}0,0 \\
0\end{array}$ & $\begin{array}{c}100 \\
19\end{array}$ & $\begin{array}{l}100 \\
16\end{array}$ & $\begin{array}{l}100, \\
14\end{array}$ \\
\hline$\%$ & 26 & 25 & 28,6 & 15,8 & 18,8 & 28,6 & 57,9 & 56,2 & 42,8 & 0,0 & 0,0 & 0,0 & 100, & 100 & 100 \\
\hline$\underset{\%}{\text { GORRO }} \mathbf{N}^{\circ}$ & $\begin{array}{l}09 \\
47 \\
4\end{array}$ & $\begin{array}{l}05 \\
31,3\end{array}$ & $\begin{array}{r}05 \\
35,7\end{array}$ & $\begin{array}{l}01 \\
5,2\end{array}$ & $\begin{array}{l}01 \\
6,2\end{array}$ & $\begin{array}{l}01 \\
7,2\end{array}$ & $\begin{array}{c}09 \\
47,4\end{array}$ & $\begin{array}{l}10 \\
62,5\end{array}$ & $\begin{array}{l}08 \\
57,1\end{array}$ & $\begin{array}{r}0 \\
0,0\end{array}$ & $\begin{array}{r}0 \\
0,0\end{array}$ & $\begin{array}{r}0 \\
0,0\end{array}$ & $\begin{array}{r}19 \\
100\end{array}$ & $\begin{array}{l}16 \\
100\end{array}$ & $\begin{array}{l}14 \\
100,\end{array}$ \\
\hline
\end{tabular}

Para os anestesistas, foram avaliados somente os componentes que eles devem utilizar durante toda a sua permanência na SO: uniforme privativo, máscara e gorro. As luvas e os óculos de proteção não permitiram sua avaliação, uma vez que são utizados em momentos diferentes do cirurgião e do instrumentador, não permanecendo com os mesmos todo o tempo. Já, os aventais e campos cirúrgicos nem sempre precisam ser utilizados pelos anestesistas.
Os componentes considerados foram utilizados por todos os anestesistas, sendo que a adequação do uniforme privativo foi total. O mesmo não ocorreu com a máscara e o gorro, já que a maioria utilizou de forma inadequada. As causas para esse alto índice de inadequação foram:

- gorro : mechas de cabelo soltas.

- máscaras : abertura nas laterais; fixação abaixo do nariz; não utilização em pelo menos um dos momentos do ato cirúrgico.

Tabela 4 - Avaliação do uso da paramentação cirúrgica pelos circulantes de SO, durante a cirurgia. São Paulo, 1998.

\begin{tabular}{|c|c|c|c|c|c|c|c|c|c|c|c|c|c|c|c|}
\hline \multirow{2}{*}{$\begin{array}{l}\text { AVAL. } \\
\text { MOMENTO } \\
\text { COMPON. }\end{array}$} & \multicolumn{3}{|c|}{ ADEQUADO } & \multicolumn{3}{|c|}{ PARC. ADEQ. } & \multicolumn{3}{|c|}{ INADEQUADO } & \multicolumn{3}{|c|}{ NÃO UTILIZOU } & \multicolumn{3}{|c|}{ TOTAL } \\
\hline & $1^{\circ}$ & $2^{\circ}$ & $3^{\circ}$ & $1^{\circ}$ & $2^{\circ}$ & $3^{\circ}$ & $1^{\circ}$ & $2^{\circ}$ & $3^{\circ}$ & $1^{\circ}$ & $2^{\circ}$ & $3^{\circ}$ & $1^{\circ}$ & $2^{\circ}$ & $3^{\circ}$ \\
\hline UNIF. No & 20 & 21 & 17 & 0 & 0 & 0 & 0 & 0 & 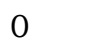 & 0 & 0 & 0 & 20 & 21 & 17 \\
\hline PRIV. \% & 100 & 100 & 100 & 0,0 & 0,0 & 0,0 & 0,0 & 0,0 & 0,0 & 0,0 & 0,0 & 0,0 & 100 & 100 & 100 \\
\hline $\begin{array}{r}\text { MÁSC. No } \\
\%\end{array}$ & $\begin{array}{l}11 \\
5,0\end{array}$ & $\begin{array}{l}09 \\
42,9\end{array}$ & $\begin{array}{l}07 \\
41,2\end{array}$ & $\begin{array}{l}02 \\
10,0\end{array}$ & $\begin{array}{r}01 \\
4,7\end{array}$ & $\begin{array}{r}01 \\
5,9\end{array}$ & $\begin{array}{l}07 \\
35,0\end{array}$ & $\begin{array}{l}11 \\
52,4\end{array}$ & $\begin{array}{l}09 \\
52,9\end{array}$ & $\begin{array}{l}0 \\
0,0\end{array}$ & $\begin{array}{c}0 \\
0,0\end{array}$ & $\begin{array}{l}0 \\
0,0\end{array}$ & $\begin{array}{l}20 \\
100\end{array}$ & $\begin{array}{c}21 \\
100\end{array}$ & $\begin{array}{r}17 \\
100\end{array}$ \\
\hline $\begin{array}{r}\text { GORRO No } \\
\%\end{array}$ & $\begin{array}{l}10 \\
50,0\end{array}$ & $\begin{array}{l}10 \\
47,6\end{array}$ & $\begin{array}{l}07 \\
41,2\end{array}$ & $\begin{array}{l}0 \\
0,0\end{array}$ & $\begin{array}{r}0 \\
0,0\end{array}$ & $\begin{array}{r}01 \\
5,9\end{array}$ & $\begin{array}{l}10 \\
50,0\end{array}$ & $\begin{array}{l}11 \\
52,4\end{array}$ & $\begin{array}{l}09 \\
52,9\end{array}$ & $\begin{array}{l}0 \\
0,0\end{array}$ & $\begin{array}{c}0 \\
0,0\end{array}$ & $\begin{array}{l}0 \\
0,0\end{array}$ & $\begin{array}{l}20 \\
100\end{array}$ & $\begin{array}{c}21 \\
100\end{array}$ & $\begin{array}{r}17 \\
100\end{array}$ \\
\hline $\begin{array}{l}\text { CAMPO No } \\
\text { CIRÚRG. \% }\end{array}$ & $\begin{array}{l}07 \\
35,0\end{array}$ & $\begin{array}{l}01 \\
4,7\end{array}$ & $\begin{array}{l}01 \\
5,9\end{array}$ & $\begin{array}{l}0 \\
0,0\end{array}$ & $\begin{array}{r}0 \\
0,0\end{array}$ & $\begin{array}{r}0 \\
0,0\end{array}$ & $\begin{array}{c}0 \\
0,0\end{array}$ & $\begin{array}{l}0 \\
0,0\end{array}$ & $\begin{array}{l}02 \\
11,7\end{array}$ & $\begin{array}{l}13 \\
65,0\end{array}$ & $\begin{array}{r}20 \\
95,3\end{array}$ & $\begin{array}{l}14 \\
82,4\end{array}$ & $\begin{array}{l}20 \\
100\end{array}$ & $\begin{array}{c}21 \\
100\end{array}$ & $\begin{array}{r}17 \\
100\end{array}$ \\
\hline
\end{tabular}

Os componentes avaliados foram uniforme privativo, máscara, gorro e campos. Os outros itens não foram avaliados por não serem usados sistematicamente por essa categoria profissional.

Somente os campos não foram usados por todos os circulantes e, aqueles que o fizeram, quase todos foram avaliados como adequados. Todos os circulantes utilizaram o uniforme privativo de forma adequada $(100,0 \%)$. O mesmo não ocorreu com a máscara t o gorro, tendo sido ambos nos três momentos, aproximadamente metade adequados e metade inadequados. As situações que determinaram a inadequação foram:

- gorro: mechas de cabelo soltas.

- máscara: abertura nas laterais; amarração apenas da fita superior; fixação abaixo do nariz.

- campo :_contato das mãos e do uniforme privativo com áreas contaminadas do campo, na sua retirada. 
A tabela a seguir permite uma melhor visualização da situação obtida de todos os profissionais ao mesmo tempo. As porcentagens apresentadas referem-se à média dos 3 momentos.

Tabela 5 - Avaliação do uso da paramentação pela equipe cirúrgica, durante a cirurgia. São Paulo, 1998.

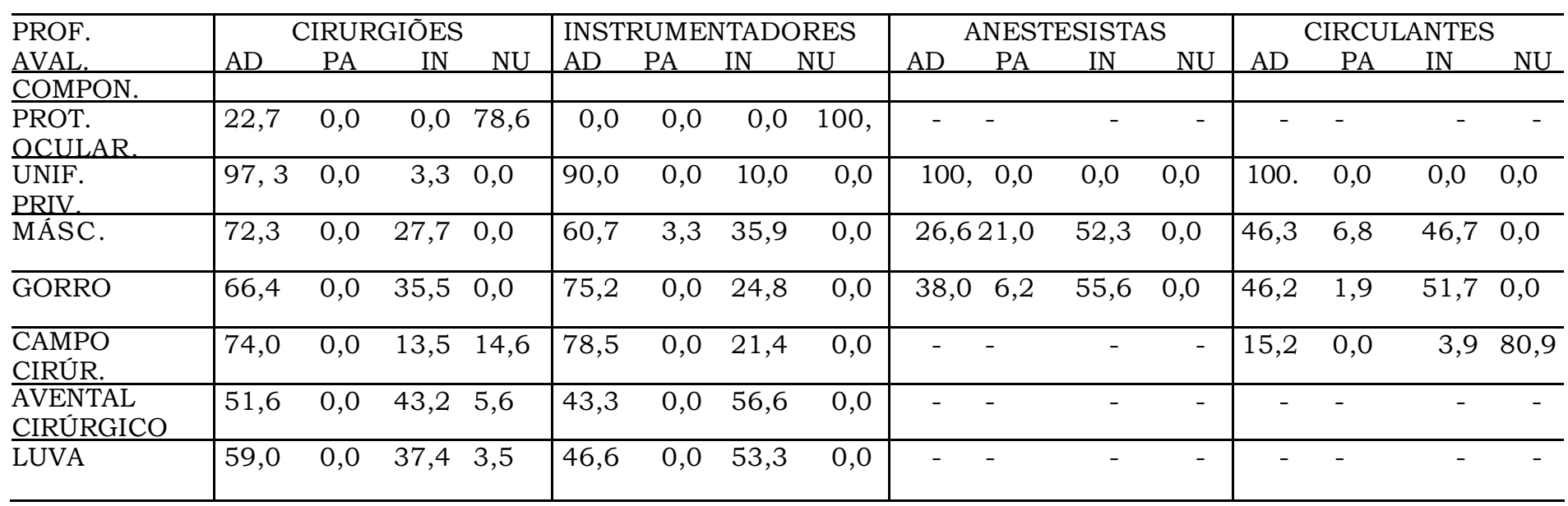

\section{AD (adequado) IN (inadequado) PA (parcialmente adequado)}

O componente utilizado de forma adequada por todos os profissionais foi o uniforme privativo. E, conforme visto, a inadequação observada refere-se a problema de confecção do mesmo (calças curtas) e não ao modo de uso pelos profissionais. Ressalte-se que nenhuma calça era fechada nos tornozelos e as mangas de vários jalecos não cobriam totalmente as axilas. $O$ protetor ocular somente foi utilizado pelos cirurgiões e, mesmo assim, pela minoria deles.

A máscara esteve adequada principalmente nos cirurgiões $(72,3 \%)$, seguidos pelos instrumentadores $(60,7 \%)$, sendo muito baixo o índice de adequação entre anestesistas $(26,6 \%)$ e baixo entre os circulantes $(46,7 \%)$. Ressalte-se que ela não foi trocada por nenhum membro da equipe em cirurgias superiores a duas horas.

O gorro esteve adequado na maioria dos instrumentadores $(75,2 \%)$ e cirurgiões $(66,4 \%)$ e inadequado na maioria dos anestesistas (56,6\%) e circulantes $(51,7 \%)$. Os campos cirúrgicos foram usados adequadamente pela maioria dos intrumentadores e cirurgiões. Não foram usados pelos anestesistas e por poucos circulantes.

O avental e as luvas foram os itens mais problemáticos entre cirurgiões e principalmente instrumentadores. Ressalte-se que as luvas não foram trocadas por nenhum deles em cirurgias superiores a duas horas, tampouco foram usadas luvas duplas.

Todos os profissionais entrevistados reconhecem a importância da paramentação como barreira contra riscos biológicos tanto para o paciente quanto para o profissional, relacionando-os com infecção hospitalar e aquelas trazidas de "fora" pelos profissionais. Já, no que se refere aos fatores que interferem na manutenção da técnica asséptica, os próprios entrevistados referiram principalmente, a má técnica cirúrgica, o fluxo de pessoas, o espaço físico da $\mathrm{SO}$, a qualidade do material utilizado e a eficiência do centro de material. Além disso, foram citados também o alto movimento cirúrgico, o tempo de cirurgia, o tipo de cirurgia e a capacitação dos profissionais.

\section{CONCLUSÃO E CONSIDERAÇÕES FINAIS}

O estudo avaliou, através de observação sistematizada, o desempenho da equipe cirúrgica na utilização da paramentação. $O$ procedimento de observação adotado foi válido, permitindo reconhecer com detalhes aspectos positivos e problemáticos. Com exceção dos óculos de proteção, a grande maioria dos profissionais utilizou todos os componentes da paramentação cirúrgica, de acordo com suas funções. A questão esteve na forma de sua utilização, a qual se distinguiu, inclusive, por categoria profissional.

Os gorros e máscaras foram mais problemáticos entre anestesistas e circulantes; o avental e a luva, entre cirurgiões e instrumentadores.

O maior índice de inadequação geral ocorreu entre instrumentadores. Isso remete à preocupação com a situação dessa categoria ocupacional, que em nosso meio, apenas recentemente foi determinado pelo COREn, a necessidade de formação básica na área de saúde, quando antes bastava realizar um curso específico, sem regulamentação. Além disso, também não está definido seu papel hierárquico no hospital, para que possam responder inclusive, a normas e treinamento. 
A causa mais freqüente para os problemas encontrados está relacionada às técnicas de assepsia, tendo sido freqüente o contato das áreas estéreis dos componentes da paramentação com pele, equipamentos e superfícies não estéreis, principalmente na colocação e retirada das luvas e dos aventais. Ao colocar as luvas, o profissional segurava-as simultaneamente, tocando as mãos no lado que deve ficar estéril. O mesmo acontecia na colocação do avental. O inverso também é verdadeiro, ou seja, ao retirar esses componentes, o lado contaminado era tocado pelos profissionais. Tais ocorrências são ainda mais significativas e referem-se antes a problemas de conhecimento de técnica asséptica do que de descaso dos profissionais, porque todos foram previamente notificados dos objetivos deste estudo. Em outras palavras, é esperado que sob essas circunstâncias, as pessoas se preocupassem mais em acertar do que errar.

Outros desempenhos inadequados talvez sejam conseqüência de desconhecimento dos modos de transmissão de infecção em cirurgia, uma vez que, pelos menos aqueles entrevistados, acreditavam na importância da paramentação como barreira. É o caso de gorros e máscaras, que em vários momentos não cobriam totalmente os cabelos, o nariz e a boca. Isso ocorria principalmente entre anestesistas e circulantes sugerindo que, por não participarem diretamente do ato cirúrgico, não necessitariam do mesmo rigor asséptico que os outros profissionais. Em menor proporção, os problemas situam-se na instituição. É o caso da qualidade dos componentes quanto à confecção e tamanho: calças curtas e sem fechamento nos tornozelos, luvas que rasgam com frequência e gorros que não conseguem sustentar todos os tipos de cabelos.

As instituições e seus profissionais necessitam rever tais questões, de tal maneira que justifiquem o uso da paramentação antes como prática que realmente seja significativa no controle de contaminações e infecções em cirurgia do que como mero ritual.

\section{REFERENCIAS BIBLIOGRÁFICAS}

1.ASSOCIATION OF OPERATION ROOM NURSING. Proposed recommended practices for uses and selection of barrier materials for surgical gowns and dropes. AORN J. , v.62, n.3, p.449-53, 1995.

2. AYLIFFE, G.A. et al. Control of hospital infection. London, Chapman \& Hall Medical. 1992. p. 211-30. cap. 11: Asepsis in operating theatres.

3. AYLIFFE, G.A.; LILLY, H. Cross-infection and its prevention. J.Hosp. Infect., n.6, p.4'7-5'7, 1985. Suppl. B.
4. BELL, D.M. et al. Preventing bloodborne pathogen transmission from healthcare worker to patients. Surg. Clin.North Am., v.75, n.5, p.1189-203, 1995.

5. BENNETT, J.V.; BRACHAMN, P.S. Hospital infections. Boston, Little Brown, 1992. p. 461-73.

6. CARTER, R. Ritual and risk. Nurs.Times, v.86, n. 13 , p.63-4, 1990 .

7. CHEN, S.K. et al. Evaluation of single-use masks and respirators for protection of health care workers against mycobacterial aerosols. Am.J.Infec.Control, v.22, n.2, p.6574, 1994.

8. COOP, G. et al. Footwear practices and operating room contamination. Nurs. Res., v.36, n.6, p.366-9, 1987.

9. EARL, A. Operating room. In : OLMSTED, R.N. (ed.) Infection control and applied epidemiology. Mosby, St. Louis 1996 . cap.95, p.95-7.

10. FRY, D.B. et al. Prevention of blood exposure. Surg.Clin.North Am., v. 75, n.6, p. 1141-57, 1995.

11. GARNER, J.S. CDC Guideline for prevention of surgical wound infections . Today's OR Nurse, v.8 , n.3, p.33-40, 1986.

12. GARNER, J.S. Guideline for isolation precautions in hospitals. Inf.Cont.Hosp.Epidem., v.17, n.1, p.50-80, 1996.

13. GAYA, H. Is it necessary for staff and visitors in an intensive care unit to wear masks, hats, gowns and overshoes? Nurs. Times, v. 86, n.13, p.63-4, 1990.

14. GERBERDING, J.L. Procedure-specific infection control for preventing intraoperative blood exposures. Am.J.Infec.Control, v.21, n.6, p.364-7, 1993.

15. GERBERDING, J.L. et al. Are universal precautions realistic? Surg.Clin.North Am., v.75, n.6, p.1091-103, 1995.

16. GOUVEIA, V.R. Embalagens para esterilização: tecidos e não tecidos. Rev. SOBECC, v.2, n.4, p. 10-13, 1997.

17. GRAZIANO, K.U. et al. Uso de propés no controle da contaminação do piso da sala de operação. In: CONGRESSO BRASILEIRO DE ENFERMAGEM, 43., Curitiba, 1991. Programa. Curitiba, ABEn, 1991, p.158.

18. GRAZIANO, K.U.; LACERDA, R.A. Paramentação cirúrgica. In: LACERDA, R.A. et al. Buscando compreender a infecção hospitalar no paciente cirúrgico. São Paulo, Atheneu, 1993. p.48-52.

19. HANEY et al. Tuberculosis makes a comeback. AORN J., v.63, n.4, p.705-15, 1996.

20. JACKSON, M. M. Tuberculosis prevention and control: the particulate respirator controversy. Today's OR Nurse, v. 16, n. 5 , p.13-9, 1994.

21. LACERDA, R.A. Prevenção de transmissão de infecção no centro cirúrgico. In: FERNANDES, A. T. et al. Infecção hospitalar e suas interfaces na área da saúde. São Paulo, Atheneu. /no prelo/

22. LACERDA, R.A. Fatores de risco relacionados ao ambiente e a limpeza da sala de operações. In: LACERDA, R.A. et al. Buscando compreender a infecção hospitalar no paciente cirúrgico. São Paulo, Atheneu, 1992. cap.8, p.115-24 
LACERDA, R.A. et al. Buscando compreender a infecção hospitalar no paciente cirúrgico. São Paulo, Atheneu, 1992. p.59-60.

23. LEVINE, A.; GODOY, M. Healthcare workers and occupational exposure to AIDS. Nurs manage. v.23 , n.1 , p.59-60,1992.

24. LEWIS Jr., R.F. et al. Epidemiology of injuries by needles and other sharp instruments. Surg.Clin.North Am., v.75, n.6, p.1105-21, 1995.

25. LYNCH, P.; WHITE, M.C. Perioperative blood contact and exposures: a comparision of incident reports and focused studies. Am.J.Infec.Control, v. 21, n.6, p.357-63, 1993.

26. MANIAN, F.A. Blood and body fluid exposures among surgeons: a survey of attitudes and perceptions five years following universal precautions. Infect.Control Hosp. Epidemiol., v.17, p.172-4, 1996.

27. MARTON, E. de S. et al. Ambiente de salas de operações: análise e avaliação de sua adequação para a prevenção de riscos biológicos em cirurgia. São Paulo, 1998. (Pesquisa de iniciação científica do Programa Institucional de Bolsas de Iniciação Científica do CNPq).

28. McCULLOUGH, E.A. Methods for determining the barrier efficacy of surgical gowns. Am.J.Infec.Control, v. 21, n.6, p.368-73, 1993.

29. NICHOLS,R.L. The operating room. In: BENNETT,J.V.; BRACHMAN,P.S. (ed.) Hospital infections. Boston, Little Brown, 1992. p. 461-73.
30. PUGLIESE, G. Blood exposure in the operating room: risk and prevention strategies. Am. J. Infec.Control., v.21, n.6, p.337-42, 1993.

31. RITTER, M.A.; MARMION, P. The exogenous sources and controls of microrganisms in the operating room. Orthop. Nurs., v.7, n.4., p.23-8, 1988.

32. RONK. Surgical patients with multiantibiotic-resistant bacteria. AORN J., v.61, n..6, p.1023-34, 1995.

33. ROY, M.C. The operating theater : special environnental area. In : Wenzel , R.P. Hospital infection. Baltimore, 1997. cap. 26.

34. SHORT, L.J.; BELL, D.M. Risk of occupational infection with blood-borne pathogens in operating and delivery room settings. Am.J.Infec.Control., v. 21, n.6, p. 343-50, 1993.

35. SIQUEIRA, L.F. de G. O meio ambiente e a infecção hospitalar. In : FERNANDES, A.T. et al. Infecção hospitalar e suas interfaces na área da saúde. São Paulo, Atheneu , 1998. / no prelo/

36. TELFORD, G.L.; QUEBBEMAN, E.J. Assessing the risk of blood exposure in the operating room. Am. J. Infec. Control., v.21, n.6, p.331-6, 1993.

37. ZINNER, N.L. How safe are your gloves? A study of protective barrier properties of gloves. AORN J., v.59, n.4, p.876-82, 1994. 


\section{INSTRUMENTO DE COLETA DE DADOS}

\section{PARTE A : caracterização do hospital}

1- Tipo de hospital:

( ) Só público ( ) Só privado ( ) Público conveniado ( ) Privado conveniado ( ) Outros.

2- Porte: () Médio ( ) Grande ( ) Extra

3- Volume de cirurgias mensais:

4- Especialidades cirúrgias:

Cirurgia observada:

Início (a partir da degermação) Término(retirada da paramentação)

\section{PARTE B : observação do uso da paramentação cirúrgica}

Momento : ( aplicar um instrumento para cada momento da cirurgia)

\begin{tabular}{|c|c|c|c|c|c|c|c|}
\hline & Cirurgião & Cirurg. Assis & Anestesista I & AnestesistaII & Instrumen. & Circulante & Outros \\
\hline $\begin{array}{l}\text { Protetor } \\
\text { Ocular }\end{array}$ & (1) $\mathrm{S} \quad(1) \mathrm{N}$ & (1) $\mathrm{S} \quad$ (1) $\mathrm{N}$ & (1) $\mathrm{S} \quad(1) \mathrm{N}$ & ( ) $\mathrm{S} \quad($ ) $\mathrm{N}$ & ( ) $\mathrm{S} \quad($ ) $\mathrm{N}$ & (1) $\mathrm{S} \quad(1) \mathrm{N}$ & ( ) $\mathrm{S} \quad(1) \mathrm{N}$ \\
\hline \begin{tabular}{|l|} 
Uniforme \\
Privativo
\end{tabular} & $\begin{array}{l}\text { () } \mathrm{S} \quad(\quad \text { I } \mathrm{N} \\
\text { () Adeq. } \\
\text { () Inadeq. } \\
\text { ()Parc.Adeq. }\end{array}$ & $\begin{array}{l}\text { ( ) S ( I N } \\
\text { ( ) Adeq. } \\
\text { ( ) Inadeq. } \\
\text { ()Parc.Adeq }\end{array}$ & $\begin{array}{l}\text { ( ) } \mathrm{S} \mathrm{(} \mathrm{I} \mathrm{N} \\
\text { ( ) Adeq. } \\
\text { ( ) Inadeq. } \\
\text { ()Parc.Adeq } \\
\end{array}$ & $\begin{array}{l}\text { ( ) S ( ) N } \\
\text { ( ) Adeq. } \\
\text { ( ) Inadeq. } \\
\text { ()Parc.Adeq } \\
\end{array}$ & $\begin{array}{l}\text { ( ) S ( ) N } \\
\text { ( ) Adeq. } \\
\text { ( ) Inadeq. } \\
\text { ()Parc.Adeq } \\
\end{array}$ & $\begin{array}{l}\text { ( ) S ( I N } \\
\text { ( ) Adeq. } \\
\text { ( ) Inadeq. } \\
\text { ()Parc.Adeq } \\
\end{array}$ & $\begin{array}{l}\text { ( ) } \mathrm{S} \text { ( I N } \\
\text { ( ) Adeq. } \\
\text { () Inadeq. } \\
\text { ()Parc.Adeq }\end{array}$ \\
\hline Máscara & $\begin{array}{l}\text { ( ) } \mathrm{S} \quad \text { () } \mathrm{N} \\
\text { ( ) Adeq. } \\
\text { ( ) Inadeq. } \\
\text { ()Parc.Adeq }\end{array}$ & $\begin{array}{l}\text { ( ) } \mathrm{S} \mathrm{()} \mathrm{N} \\
\text { () Adeq. } \\
\text { () Inadeq. } \\
\text { ()Parc.Adeq }\end{array}$ & $\begin{array}{l}\text { () } \mathrm{S} \text { ( ) } \mathrm{N} \\
\text { () Adeq. } \\
\text { ( ) Inadeq. } \\
\text { ()Parc.Adeq }\end{array}$ & $\begin{array}{l}\text { ( ) } \mathrm{S} \quad \text { () } \mathrm{N} \\
\text { () Adeq. } \\
\text { () Inadeq. } \\
\text { ()Parc.Adeq }\end{array}$ & $\begin{array}{l}\text { () } \mathrm{S} \text { ( ) N } \\
\text { () Adeq. } \\
\text { () Inadeq. } \\
\text { ()Parc.Adeq }\end{array}$ & $\begin{array}{l}\text { ( ) } \mathrm{S} \mathrm{()} \mathrm{N} \\
\text { () Adeq. } \\
\text { ( ) Inadeq. } \\
\text { ()Parc.Adeq }\end{array}$ & $\begin{array}{l}\text { ( ) } \mathrm{S} \mathrm{()} \mathrm{N} \\
\text { () Adeq. } \\
\text { ( ) Inadeq. } \\
\text { ()Parc.Adeq }\end{array}$ \\
\hline Gorro & $\begin{array}{l}\text { ( ) } \mathrm{S} \text { () } \mathrm{N} \\
\text { ( ) Adeq. } \\
\text { ( ) Inadeq. } \\
\text { ()Parc.Adeq }\end{array}$ & $\begin{array}{l}\text { ( ) } \mathrm{S} \mathrm{()} \mathrm{N} \\
\text { () Adeq. } \\
\text { () Inadeq. } \\
\text { ()Parc.Adeq }\end{array}$ & $\begin{array}{l}\text { () } \mathrm{S} \text { () } \mathrm{N} \\
\text { () Adeq. } \\
\text { ( ) Inadeq. } \\
\text { ()Parc.Adeq }\end{array}$ & $\begin{array}{l}\text { () } \mathrm{S} \text { () } \mathrm{N} \\
\text { () Adeq. } \\
\text { () Inadeq. } \\
\text { ()Parc.Adeq }\end{array}$ & $\begin{array}{l}\text { () } \mathrm{S} \text { ( ) } \mathrm{N} \\
\text { () Adeq. } \\
\text { () Inadeq. } \\
\text { ()Parc.Adeq }\end{array}$ & $\begin{array}{l}\text { ( ) } \mathrm{S} \mathrm{()} \mathrm{N} \\
\text { () Adeq. } \\
\text { ( ) Inadeq. } \\
\text { ()Parc.Adeq }\end{array}$ & $\begin{array}{l}\text { ( ) } \mathrm{S} \mathrm{()} \mathrm{N} \\
\text { () Adeq. } \\
\text { () Inadeq. } \\
\text { () Parc. Adeq }\end{array}$ \\
\hline \begin{tabular}{|l|} 
Campo \\
Cirúrgico.
\end{tabular} & $\begin{array}{l}\text { ( ) } \mathrm{S}(\text { I N } \\
\text { () Adeq. } \\
\text { ( ) Inadeq. } \\
\text { ()Parc.Adeq } \\
\end{array}$ & $\begin{array}{l}\text { ( ) S ( I N } \\
\text { ( ) Adeq. } \\
\text { () Inadeq. } \\
\text { ()Parc.Adeq. }\end{array}$ & $\begin{array}{l}\text { ( ) } \mathrm{S} \mathrm{(} \mathrm{I} \mathrm{N} \\
\text { ( ) Adeq. } \\
\text { ( ) Inadeq. } \\
\text { ()Parc.Adeq }\end{array}$ & $\begin{array}{l}\text { ( ) S ( I N } \\
\text { ( ) Adeq. } \\
\text { ( ) Inadeq. } \\
\text { ()Parc.Adeq }\end{array}$ & $\begin{array}{l}\text { ( ) S ( I N } \\
\text { ( ) Adeq. } \\
\text { ( ) Inadeq. } \\
\text { ()Parc.Adeq }\end{array}$ & $\begin{array}{l}\text { ( ) S ( I N } \\
\text { ( ) Adeq. } \\
\text { ( ) Inadeq. } \\
\text { ()Parc.Adeq }\end{array}$ & $\begin{array}{l}\text { ( ) S ( I N } \\
\text { ( ) Adeq. } \\
\text { () Inadeq. } \\
\text { ()Parc.Adeq }\end{array}$ \\
\hline \begin{tabular}{|l|} 
Avental \\
Cirúrgico
\end{tabular} & $\begin{array}{l}\text { ( ) } \mathrm{S} \mathrm{()} \mathrm{N} \\
\text { () Adeq. } \\
\text { () Inadeq. } \\
\text { ()Parc.Adeq }\end{array}$ & $\begin{array}{l}\text { () } \mathrm{S} \mathrm{()} \mathrm{N} \\
\text { () Adeq. } \\
\text { () Inadeq. } \\
\text { ()Parc. Adeq. }\end{array}$ & $\begin{array}{l}\text { ( ) } \mathrm{S} \quad \text { ( ) } \mathrm{N} \\
\text { ( ) Adeq. } \\
\text { ( ) Inadeq. } \\
\text { ()Parc.Adeq }\end{array}$ & $\begin{array}{l}\text { ( ) } \mathrm{S} \mathrm{()} \mathrm{N} \\
\text { () Adeq. } \\
\text { () Inadeq. } \\
\text { ()Parc.Adeq }\end{array}$ & $\begin{array}{l}\text { () } \mathrm{S} \text { ( ) N } \\
\text { () Adeq. } \\
\text { () Inadeq. } \\
\text { ()Parc.Adeq } \\
\end{array}$ & $\begin{array}{l}\text { ( ) } \mathrm{S} \mathrm{()} \mathrm{N} \\
\text { () Adeq. } \\
\text { ( ) Inadeq. } \\
\text { ()Parc.Adeq }\end{array}$ & $\begin{array}{l}\text { () } \mathrm{S} \mathrm{()} \mathrm{N} \\
\text { () Adeq. } \\
\text { () Inadeq. } \\
\text { ()Parc.Adeq } \\
\end{array}$ \\
\hline \begin{tabular}{|l|} 
Luva \\
Cirúrgica
\end{tabular} & $\begin{array}{l}\text { () } \mathrm{S} \quad \text { () } \mathrm{N} \\
\text { () Adeq. } \\
\text { () Inadeq. } \\
\text { ()Parc.Adeq } \\
\end{array}$ & $\begin{array}{l}\text { ( ) } \mathrm{S} \mathrm{()} \mathrm{N} \\
\text { () Adeq. } \\
\text { () Inadeq. } \\
\text { ()Parc. Adeq. }\end{array}$ & $\begin{array}{l}\text { ( ) } \mathrm{S} \quad \text { ( ) N } \\
\text { ( ) Adeq. } \\
\text { ( ) Inadeq. } \\
\text { ()Parc.Adeq }\end{array}$ & $\begin{array}{l}\text { ( ) } \mathrm{S} \quad \text { () } \mathrm{N} \\
\text { () Adeq. } \\
\text { () Inadeq. } \\
\text { ()Parc.Adeq }\end{array}$ & $\begin{array}{l}\text { ( ) } \mathrm{S} \quad \text { ( ) N } \\
\text { () Adeq. } \\
\text { () Inadeq. } \\
\text { ()Parc.Adeq }\end{array}$ & $\begin{array}{l}\text { () } \mathrm{S} \text { () } \mathrm{N} \\
\text { () Adeq. } \\
\text { () Inadeq. } \\
\text { ()Parc.Adeq } \\
\end{array}$ & $\begin{array}{l}\text { () } \mathrm{S} \quad \text { () } \mathrm{N} \\
\text { () Adeq. } \\
\text { () Inadeq. } \\
\text { ()Parc.Adeq }\end{array}$ \\
\hline \multicolumn{8}{|l|}{ Anotações } \\
\hline & & & & & & & \\
\hline
\end{tabular}

